\title{
Automation Upgrade via PLC Applications for Big Harbor Delivery Facilities
}

\author{
M. Papoutsidakis \\ Dept. of Automation Eng. \\ Piraeus University \\ Of Applied Sciences, \\ Athens, Greece
}

\author{
A. Chatzopoulos \\ Dept. of Automation Eng. \\ Piraeus University \\ Of Applied Sciences, \\ Athens, Greece
}

\author{
E. Symeonaki \\ Dept. of Automation Eng. \\ Piraeus University \\ Of Applied Sciences, \\ Athens, Greece
}

\begin{abstract}
This paper deals with the improvement of a harbour wheat delivery line with the aim of automating the procedure. Specifically, this improvement will be implemented with the use of PLC and the function will be explained during the designing and the trials. In conclusion the paper is completed commenting on the results and suggesting future solutions with the aim of improving the aforementioned facility.
\end{abstract}

\section{Keywords}

Watermarking, Haar Wavelet, DWT, PSNR

\section{INTRODUCTION}

In this dissertation there will be no need to mention different types of unloading. We shall deal only with unloading ways connected with bulk cargoes. The ways are two: either with the use of a pylon which bears a special high-power vacuum pump in its functional circuit, or with the use of a special crane truck which bears a bucket in the form of a claw which can bind a big quantity of the product and release it in the trailer of a vehicle or even in a special sort of machine in the form of a funnel responsible for the loading of cargo trucks (filler funnel).

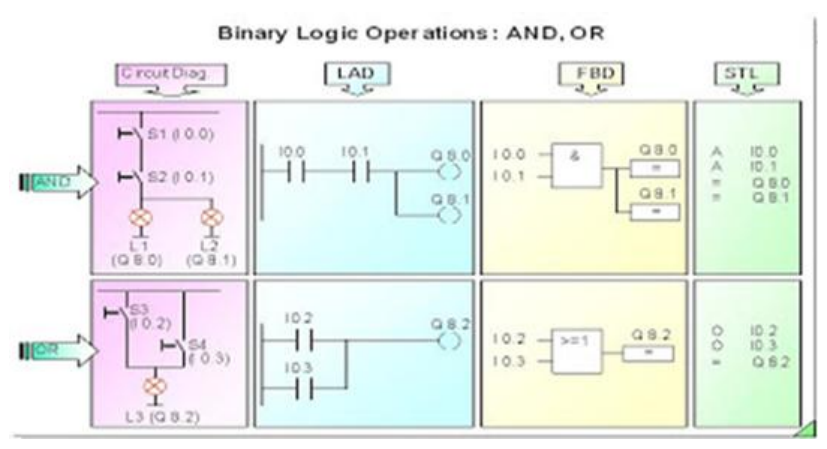

Fig 1. Binary Logic Operations: AND, OR

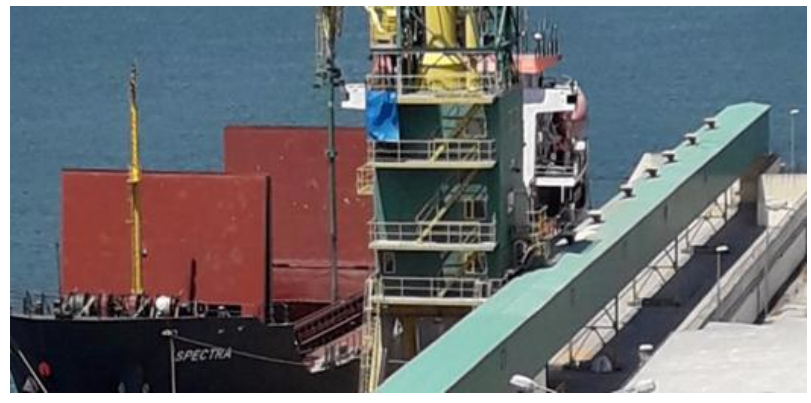

Fig 2. The unloading way

This is the procedure that the company follows too, as it owns harbour facilities. The role of the entrance trolley is most important since, as has already been mentioned, it determines the position of the boom. The position of the boom is important in the unloading procedure as it determines the spot at which the ship unloads and this spot often has to be transferred during the unloading.

\section{LOGISTICAL DESCRIPTION OF THE REQUIRED EQUIPMENT}

\subsection{Electrical relay}

The term electrical relays applies to remote control power switches whose use allows, apart from the on-spot, also the remote controlling of circuits that require connections and disconnections of high power consumption loads, in other words high amperage.

\subsection{Thermal}

When the motors start, they draw power from the circuit which is a lot of times higher than its nominal power; in many cases six or even seven times that. If we used common fuses, it wouldn't be possible to start the motor because they would interrupt its electrical supply due to the large starting current surges alone. Therefore fusions provide protection only against short circuits. For the complete protection of the motor we use slow burn fuses for the short circuits and thermal for overload cases.

\subsection{Terminal position switches}

They vary in application, function wiring type and accuracy but the general use of their application is to signify the presence of the position of some element in the installation whether that is from some open blockage drawer, or some confirmation of feedback response or even the filling and unloading of a tank or a conveyor belt. Because they can determine the starting or even the cease of an automatic function they' re also known as limit switches.

\subsection{Terminal detection switches / proximity sensors}

These switches are also called proximity sensors. They're electronic, follow a dyadic logic and are activated before they even come in contact with the detectable object and thus we also call them inductive.

\subsection{Push buttons}

Push buttons are a kind of button that is used in automation installations, although they display a distinctiveness in their functional application. Pressing the key of the button we need to keep in mind that it has a return spring, so the activation order lasts for as long as the key is pressed. [see 9] 


\subsection{Switches}

Switches are mechanisms that allow the connection and disconnection of electrical power in an installation or mechanism. Apart from the cargo switches which have been designed for disconnections of high voltage it is good to take a closer look at a category of switches called emergency stop switches.

\subsection{Timers}

In applications of automatic control and handling there's frequently a need for delay either in the turning on or in the turning off of the operation of the circuit.

1. Delay in the activation or delay on

2. Delay in the deactivation or delay off

3. Pulse time operation which depends on the length of supply, also known as one shot

4. Pulse time operation which is unrelated to the length of supply also known as interval. [see 9]

\section{THE OLD WAY OF IMPLEMENTING THE APPLICATION}

What we want this application to achieve is to move the wheat unloading trolley, depending on its position, to a belt or right position into a star or delta at the starting of the motor. The problem to date has been that the pylon operator needs to leave the pylon and walk for about fifteen minutes to reach the area where the trolley is positioned in order to move the trolley to the desired position, then return to the pylon and continue the unloading procedure in case nobody else can move the trolley. Therefore, the procedure is delayed and, if the unloading takes place in the evening, we also run the risk of an accident. Therefore, the procedure requires automation which will help save time and assure the smooth running of the procedure minimizing the risk of an accident for the operator as well.

\subsection{Criteria for choosing a way to start the motor}

For this reason which primarily depends on the motor power, one of the following ways to start is chosen:

1. Immediate start which is recommended for motors under $0,75 \mathrm{KW}$

2. Start with resistance in the stator which is recommended for high power applications

3. Start with a star delta wiring either with a switch or automation

4. Start with controlling the speed of the motor through frequency - inverter which is the smoothest way and ascertains the longest lifespan in motors.

For this application a motor already exists and the way that has been chosen to start it is through star delta automation as it is more cost-effective in relation to the other motors available.

\subsection{Motor start with an automatic star delta switch with reversal}

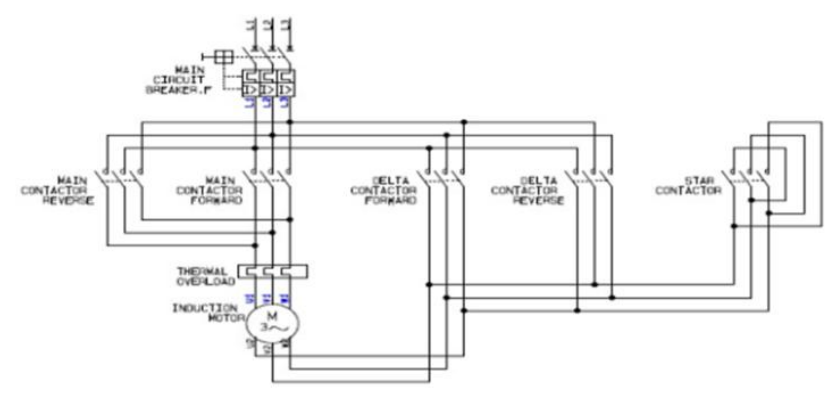

Fig. 3. The power circuit of the old application

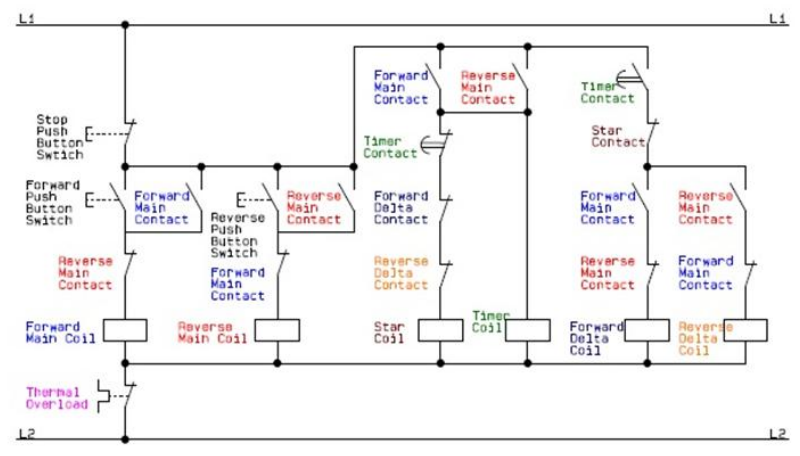

Fig. 4: The auxiliary circuit of the application But to make the description clearer we need to specify the fact that there are two independent circuits:

1. The power circuit which is related to the power currents transient through the main contacts of the relays.

2. The auxiliary circuit which is related to the auxiliary currents which allow us the handling and indications in an application.

Once the direction is activated and the motor has been activated through the star and has acquired the power necessary to switch to its rated speed / capacity and in due time (which has been determined by us) the star relay is deactivated, the main conductor coil relay remains active as well as the direction relay, while simultaneously the delta relay is activated so that the motor can achieve its rated speed. [see 1]

\subsection{Logical gates}

AND: This gate is termed AND because, in order for it to have the logic output one, all its inputs need to have a logic one. Any other combination of inputs will have an output zero.

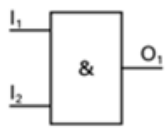

$$
O_{1}=I_{1} \cdot I_{2}
$$

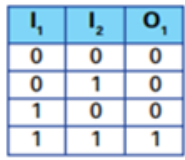

Fig. 5. Logical gate AND

OR: This gate is termed OR because, in order for it to have the output one, at least one or all of its inputs need to have a logic one. 


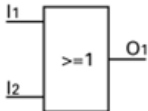

$$
O_{1}=I_{1}+I_{2}
$$

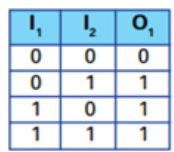

Fig. 6. Logical gate $O R$

NOT: This gate implements logical negation as it has the tendency to transform all its input to the exact opposite.

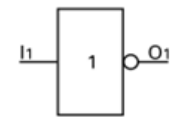

$$
\mathbf{O}_{1}=\bar{T}_{1}
$$

Fig. 7. Logical gate NOT

XOR: The function of this gate is based on the Exclusive OR that is for it to have a logic output 1, only one of its inputs must have logic 1 . Anything other than that results in a zero logic output [see 4].
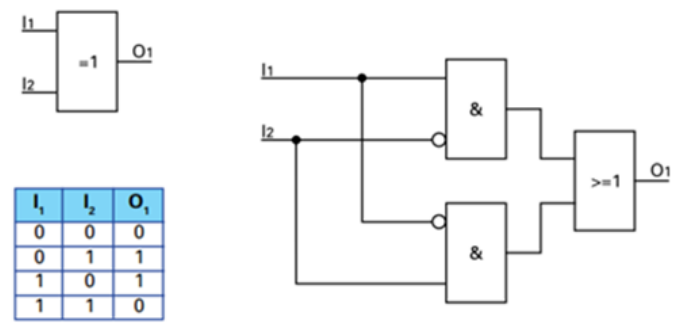

Fig. 8. Logical gate XOR.

\section{IMPLEMENTATION - IMPROVEMENT WITH PLC}

For the implementation of the application LOGO! Soft Comfort software by Siemens was used. After pressing twice on the icon, the operator is introduce to the software environment. File $=>$ New $=>$ Function Block Diagram needs to be chosen on the top left, that is FBD programming language was used to implement the project. Now the operator is ready to write the code. By choosing the fourth icon on the left vertical bar (the icon $\mathrm{CO}$ ), a range of choices is opened on the bottom of the screen. Pressing on the first one, which bears the symbol I, input can be entered and by pressing twice on it a window of three choices opens. The first one is termed "Parameter" and it determines which input it is, the second is termed "Comment" and allows you to comment on the input and the third one, "Simulation", determines the behaviour of the input that is whether it is a close or open button, a switch. After GF key is chosen on the lower desktop, a horizontal command bar opens. The first choice on this horizontal bar, bearing the symbol " $\&$ " is the AND gate. The introduction of OR was implemented by choosing GF on the left vertical bar of choices, and from the list the fifth icon with the symbol $\leq 1$ was chosen. This gate is B005 and has a logic output 1 if one of its inputs has a logic 1 . The part that provides the user with a special function, bears two inputs: SET which, when its input is logic 1 , logic 1 appears in the output which is selfretained, and RESET which, once triggered with a logic 1, impels the output to zero (0). In order for this function to be introduced to the programme, the icon SF (Special Functions) needs to be choosen on the left vertical bar of tool choices.

By choosing SD, a new horizontal bar of choices appears on the bottom of the desktop which pertains to SET - RESET. In order for the timer to be introduced to the programme, the sixth key with the symbol SF. must be pressed and it will open a horizontal command list at the bottom from which we need to choose the first command "delay on". By choosing the "delay on" key the timer appears on the screen. By tapping twice on the timer, a configuration window opens in which the timer regulation time through the "parameter" choice is determined, whereas through the "Comment" choice, commenting takes place. [see 6-7]

\subsection{Clockwise movements}

\subsubsection{Step 1: From position 1 to position 2}

The prerequisites for the trolley to move from position 1 to position 2 is the call from the press button I12 POSITION 2 CALL and definitely the signal from the sensor I1 POSITION PARK 1 until the trigger of sensor I2 POSITION PARK 2 which simply means that the wheat input carriage has reached position 2 from position 1 . The I7 and I8 are normally NC contacts in the wiring so they will have logic 1 in inactive condition and while activated they will have logic zero and the reason is as commanded in Q1 output which is responsible for the right movement of the carriage from position 1 to position 2 .

\subsubsection{Step 2: From position 1 to position 3}

Sensor I1 must command POSITION 1 PARK. I1 must be combined with an AND gate, B002, with the input I13 which is the transition call from position 1 to position 3 with the use of press buttons. The stopping can occur either from I7 I8 as in the movement above or by triggering the sensor position 3 I3.

\subsubsection{Step 3: From position 1 to position 4} Sensor I1 must command POSITION 1 PARK. I1 must be combined with an AND gate, with the input I14 which is the transition call from position 1 to position 3 with the use of press buttons. The stopping can occur either from I7 I8 as in the movement above or by triggering the sensor position 4 I4.

\subsubsection{Step 4: From position 2 to position 3}

Sensor I2 must command POSITION 2 PARK. I1 must be combined with an AND gate, with the input I13 which is the transition call from position 1 to position 3 with the use of press buttons. The stopping can occur either from I7 I8 as in the movement above or by triggering the sensor position $4 \mathrm{I}$. [see 6-7]

\subsubsection{Step 5: From position 3 to position 4}

In the present phase AND B012 takes command from the choice I5 POSITION 4 CALL and the sensor needs to see POSITION 2 PARK for the carriage to start moving to position 4. AND B012 gives the command "Set" to Set Reset B013 and its output gives a command to OR B014. "Reset" is received by sensor I4 POSITION 4 PARK

\subsubsection{Step 6: from position 3 to position 4}

In that case, for the wheat input carriage to move from position 3 to position 4, gate AND B015 needs to have input from I5, that is POSITION 4 CALL, and from I3, which is position 3 PARK.

As far as these two conditions are met, AND B006 gives a command to Q1 exit responsible for the right (clockwise) movements and it will stop on its own from I7, I8, I4.

\subsection{Counterclockwise movements}

\subsubsection{Step 7: From position 4 to position 1}

Gate AND B017 takes command from I11 which is a call command to position 1 and from I4 which is presence command for the carriage in position 4. The output of AND B017 supplies "Set" of B019 exactly as applied to clockwise 
movements, and supplies Q2 left movement exit. The SetReset B019 is deactivated from I1, which means the carriage has reached position 1 .

\subsubsection{Step 8: From position 4 to position 2}

Gate AND B017 takes command from I11 which is a call command to position 1 and from I4 which is presence command for the carriage in position 4. The output of AND B017 supplies "Set" of B019 exactly as applied to clockwise movements, and supplies Q2 left movement exit. The SetReset B019 is deactivated from I2, which means the carriage has reached position 2 .

\subsubsection{Step 9: From position 4 to position 3}

Gate AND B023 takes command from I13 which is a call command to position 1 and from I4 which is presence command for the carriage in position 4. The output of AND B017 supplies "Set" of B020 exactly as applied to clockwise movements, and supplies Q2 left movement exit. The SetReset B019 is deactivated from I3, which means the carriage has reached position 3. [see 6-7]

\subsubsection{Step 10: From position 3 to position 2}

When gate AND B028 must have logic 1 from input I3 for presence of the carriage in position 3 PARK and I11 call command of the carriage in position 1 , it will display logic 1 in its output, which will give command SET of Set - Reset B022 which will activate its output by supplying gate OR B023 which will activate Q2 for as long as it takes for the carriage to reach position 2 by activating Reset of B022 triggering $\mathrm{I} 2$.

\subsubsection{Step 11: From position 3 to position 1}

When gate AND B028 must have logic 1 from input I3 for presence of the carriage in position 3 PARK and I11 call command of the carriage in position 1, it will display logic 1 in its output, which will give command SET of Set - Reset B026 which will activate its output by supplying Q2 command left course for as long as logic 1 in Reset of B026 unless course is interrupted from sensor I1. [see 6-7]

\subsubsection{Step 12: From position 2 to position 1}

When gate AND B028 must have logic 1 from input I2 for presence of the carriage in position 2 PARK and I11 call command of the carriage in position 1, it will display logic 1 in its output, which will give command SET of Set - Reset B031 which will activate its output by supplying Q2 command left course for as long as logic 1 in Reset of B026 unless course is interrupted from sensor I1. [see 6-7]

\subsubsection{Step 13: Star delta}

Gate XOR was chosen because its output must be exclusively activated for either right or left movement, as this gate is activated for one only of the outputs, and disallow the simultaneous function of star and delta. So whichever of the two inputs acquires logic 1 will supply a XOR input which will activate its output, which will activate SET of Set - Reset B029 and at the same time timer B032 the SET of B029 immediately the output of star Q3 is activated and in the timer in reverse to count the time that has been set. After the set time passes, timer B032 is activated and it immediately triggers Reset of B029 and so Q3 star automatically stops and Q4 delta starts. [see 6-7]

\section{PROGRAMME TRIALS}

A procedure which is as important as the design which was explained in the previous unit, is the trial procedure, in that it defines whether the designed programme is stable and whether it meets the conditions for which it has been designed.After Simulink is activated in the lower part of the desktop a horizontal bar of choices appears which is different from the previous ones used during the designing phase. On the left side, up to the middle of the bar the inputs are distinctive because they bear the term "I". After the inputs, it is obvious in order of appearance the line with the outputs which are symbolised with the bulbs. Q is known as output and $\mathrm{M}$ as auxiliary memory. Inline after the outputs the symbol power is obvious which shows that PLC is activated and able to simulate the lack of voltage, while after that the green horizontal arrow follows which activates the programme, and if we drag the mouse cursor on this the term RUN appears. As long as there is RUN there is also STOP which is the next choice inline and when it is available is coloured red. The next choice if the mouse cursor is dragged on, shows the term suspend / resume simulation and when run is activated, suspend assumes a yellow colour and it is available to use to temporarily pause the function without general interruption and if it is activated again, the programme can resume from where is stopped before the pause. The trials where applied first on the right movements and then on the left movements and after that on the star-delta switchover.

\section{CONCLUSIONS - IMPROVEMENTS}

Based on the above it was determined that the automation programme for the procedure is implementable and beneficial, as during the trials it was made obvious that it can be smoothly implemented and it has clear benefits. The programme obviously improves the procedure by minimizing loss of time and by also rendering the work of the operator safer as the person doesn't need to leave the pylon to change positions. As far as improvements are concerned, both the use of more terminal sensors and the use of inverter when the hours of use are increased, will improve the response to call command as well as the lifespan of the motor.

\section{ACKNOWLEDGMENTS}

All authors would like to express their gratitude to the PostGraduate Program of Studies "Automation of Productions and Services" of PUAS, for the financial support to undertake this research project 


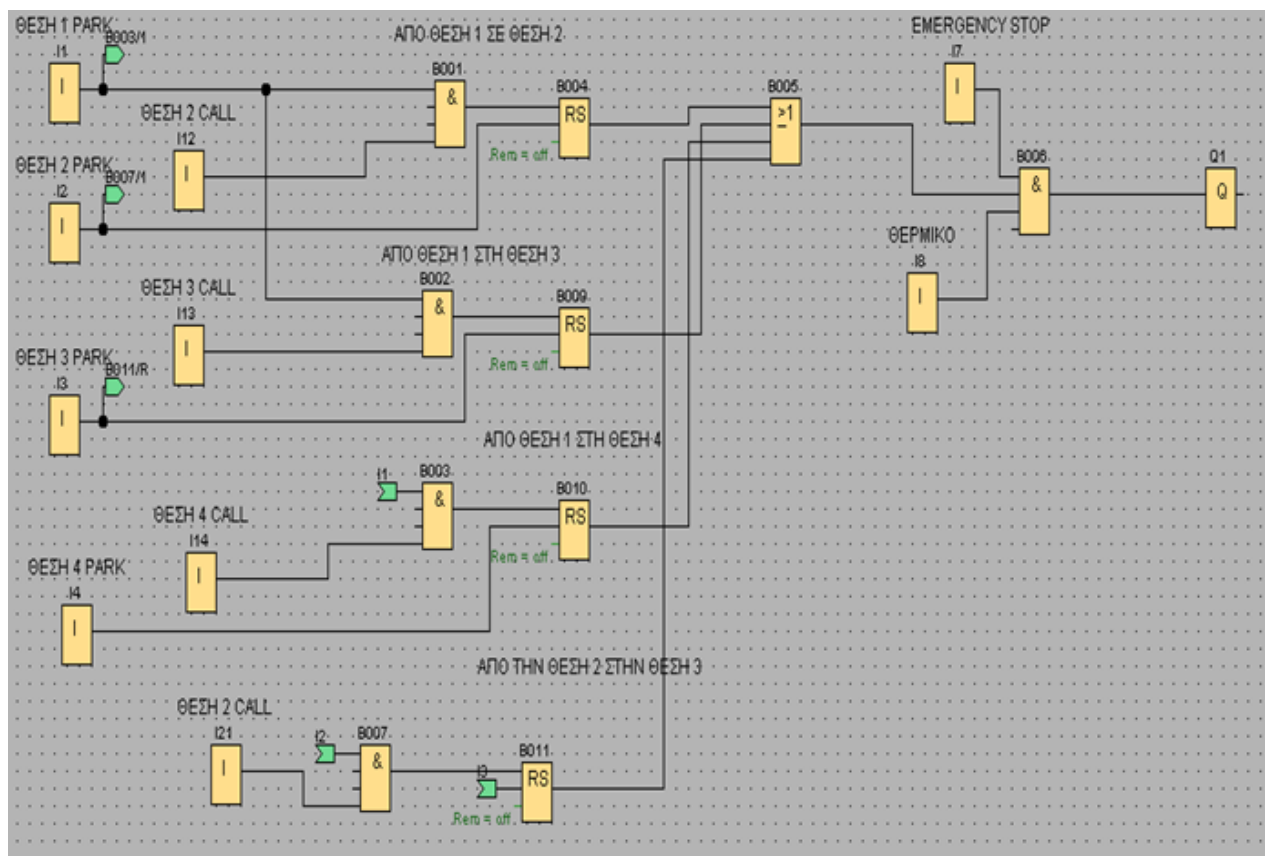

Fig. 9. Offline part of program

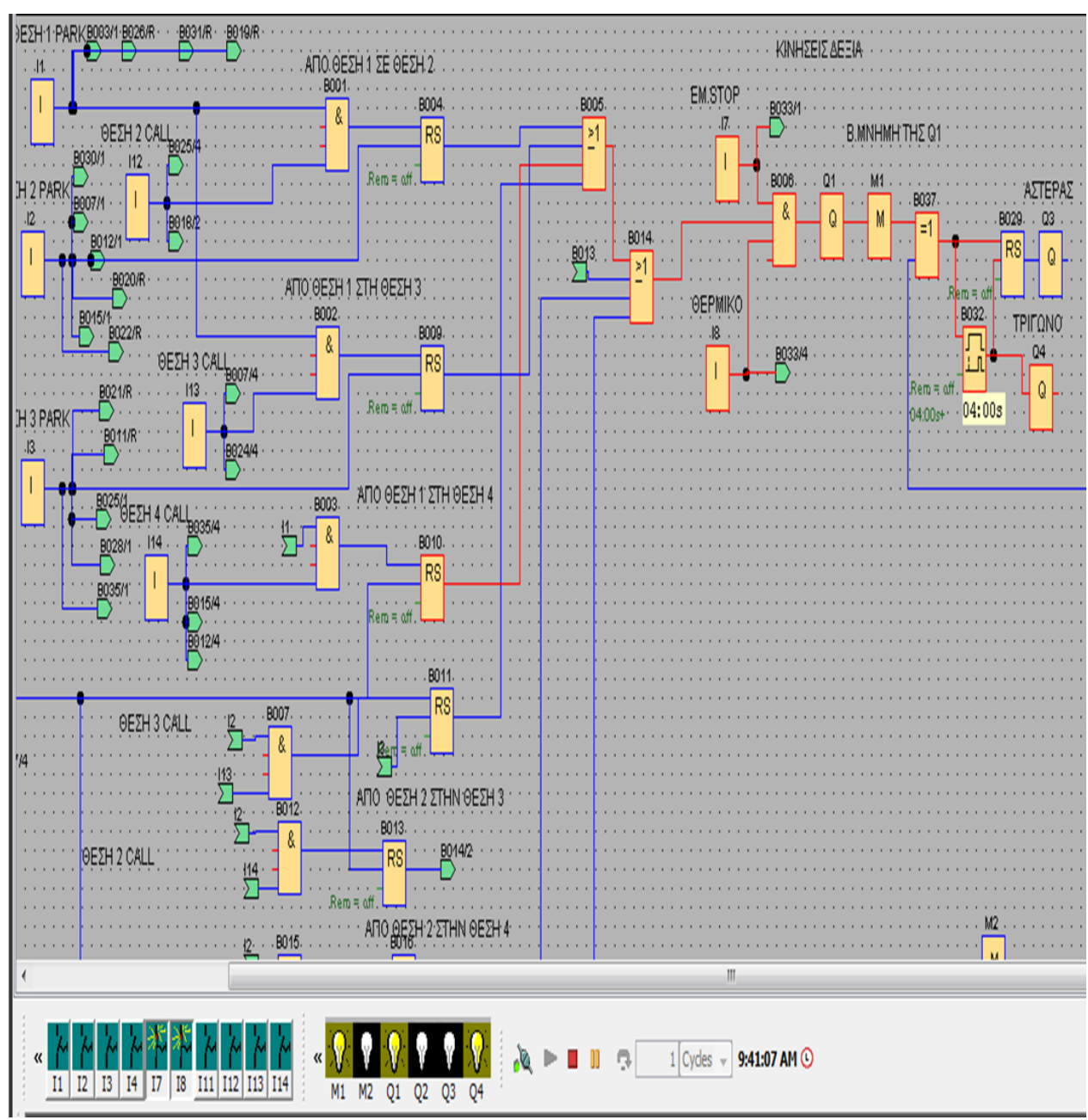

Fig. 10. Online monitoring of program 


\section{REFERENCES}

[1] Bowman, M., Debray, S. K., and Peterson, L. L. 1993. Reasoning about naming systems. .

[2] Ding, W. and Marchionini, G. 1997 A Study on Video Browsing Strategies. Technical Report. University of Maryland at College Park.

[3] Fröhlich, B. and Plate, J. 2000. The cubic mouse: a new device for three-dimensional input. In Proceedings of the SIGCHI Conference on Human Factors in Computing Systems

[4] Tavel, P. 2007 Modeling and Simulation Design. AK Peters Ltd.

[5] Sannella, M. J. 1994 Constraint Satisfaction and Debugging for Interactive User Interfaces. Doctoral
Thesis. UMI Order Number: UMI Order No. GAX9509398., University of Washington.

[6] Forman, G. 2003. An extensive empirical study of feature selection metrics for text classification. J. Mach. Learn. Res. 3 (Mar. 2003), 1289-1305.

[7] Brown, L. D., Hua, H., and Gao, C. 2003. A widget framework for augmented interaction in SCAPE.

[8] Y.T. Yu, M.F. Lau, "A comparison of MC/DC, MUMCUT and several other coverage criteria for logical decisions", Journal of Systems and Software, 2005, in press.

[9] Spector, A. Z. 1989. Achieving application requirements. In Distributed Systems, S. Mullender 